EPJ Web of Conferences 53, 06002 (2013)

DOI: $10.1051 /$ epjconf/20135306002

(C) Owned by the authors, published by EDP Sciences, 2013

\title{
Transition from Galactic to extragalactic cosmic rays and cosmic ray anisotropy
}

\author{
G. Giacinti ${ }^{1}$, M. Kachelrieß ${ }^{1}$, D.V. Semikoz ${ }^{2, a}$ and G. Sigl $\left.\right|^{3}$ \\ ${ }^{1}$ IInstitutt for fysikk, NTNU, Trondheim, Norway \\ ${ }^{2}$ AstroParticle and Cosmology (APC), Paris, France \\ ${ }^{3}$ Institut für Theoretische Physik, Universität Hamburg, Germany
}

\begin{abstract}
This talk based on results of ref. [1], where we constrain the energy at which the transition from Galactic to extragalactic cosmic rays occurs by computing the anisotropy at Earth of cosmic rays emitted by Galactic sources. Since the diffusion approximation starts to loose its validity for $E / Z \gtrsim 10^{(16-17)} \mathrm{eV}$, we propagate individual cosmic rays using Galactic magnetic field models and taking into account both their regular and turbulent components. The turbulent field is generated on a nested grid which allows spatial resolution down to fractions of a parsec. If the primary composition is mostly light or intermediate around $E \sim 10^{18} \mathrm{eV}$, the transition at the ankle is ruled out, except in the unlikely case of an extreme Galactic magnetic field with strength $>10 \mu \mathrm{G}$. Therefore, the fast rising proton contribution suggested by KASCADE-Grande data between $10^{17} \mathrm{eV}$ and $10^{18} \mathrm{eV}$ should be of extragalactic origin. In case heavy nuclei dominate the flux at $E>10^{18} \mathrm{eV}$, the transition energy can be close to the ankle, if Galactic cosmic rays are produced by sufficiently frequent transients as e.g. magnetars.
\end{abstract}

\section{INTRODUCTION}

The question at which energy the transition from Galactic to extragalactic cosmic rays (CRs) takes place is one of the major unresolved issues of cosmic ray physics. Two promising possibilities are to associate the transition with one of the two evident features of the cosmic ray spectrum: The second knee around $E \simeq 5 \times 10^{17} \mathrm{eV}$ or the ankle at $E \simeq 3 \times 10^{18} \mathrm{eV}$. Since the chemical composition of galactic and extragalactic CRs should differ in general, both because of propagation effects and of the different nature of their sources, the transition may be detected experimentally studying the chemical composition of CRs as function of energy.

In the case of a transition around the second knee, Galactic CR sources such as e.g. supernova remnants would accelerate CRs up to the rigidity-dependent knee, which is close to $10^{17} \mathrm{eV}$ for iron. If the extragalactic CR flux dominating at higher energies would consist mainly of protons, the ankle could be explained as a dip in the extragalactic CR spectrum due to the pair-production losses of protons on cosmic microwave background (CMB) photons $p+\gamma_{\mathrm{CMB}} \rightarrow p+e^{+}+e^{-}[2]$. Below $\sim 10^{17-18} \mathrm{eV}$, the extragalactic CR flux may be suppressed because of $\mathrm{CR}$ propagation in extragalactic magnetic fields [3, 4]. On the other hand, the scenario of Ref. [5] would favour a transition at the ankle. The composition of the CR flux at high energies is the subject of current debate due to the facts that hadronic physics must be extrapolated from lower energies and that the complex experimental analyses for different experiments are not yet completely reconciled. The scenario of Ref. [2] is supported by the composition measurements of HiRes [6] and the first results of the Telescope Array [7], which are

\footnotetext{
${ }^{a}$ e-mail: dmitri.semikoz@apc.univ-paris7.fr
}

This is an Open Access article distributed under the terms of the Creative Commons Attribution License 2.0, which permits unrestricted use, distribution, and reproduction in any medium, provided the original work is properly cited. 
consistent with a light composition around the ankle and above. On the other hand, recent results from the Pierre Auger Observatory [8, 9] indicate a composition becoming heavier with increasing energy above the ankle, and the Yakutsk EAS array muon data suggests a non negligible fraction of heavy nuclei above $\simeq 10^{19} \mathrm{eV}$ [10]. Moreover, the measurements of the KASCADE-Grande [11] collaboration are consistent with a dominantly heavy composition up to $10^{18} \mathrm{eV}$. However, the KASCADE-Grande data indicate a fast rising proton contribution above $10^{17} \mathrm{eV}$.

Thus at present the experimental data on the CR composition do not allow us yet to determine the transition energy between Galactic and extragalactic CRs. In this paper we suggest to use instead experimental limits on the anisotropy of the arrival directions of UHECRs to constrain the maximal contribution of Galactic CRs at $E \gtrsim 10^{18} \mathrm{eV}$. At energies below $10^{17} \mathrm{eV}$, the diffusive propagation of Galactic cosmic rays and their resulting anisotropy at Earth was studied in details in Refs. [12, 13].

Since the propagation of CRs in the Galactic magnetic field (GMF) is not longer diffusive at $E \gtrsim 10^{17} \mathrm{eV}$, in ref. [1] we directly propagated UHECRs in the GMF using the numerical code developed in Refs. $[14,15]$. Also we presented also a way to generate the turbulent field on a nested grid without limitation on its spatial resolution. This method allowed us to include magnetic field fluctuations spanning the required large dynamical range of scales, from negligible compared to the CR Larmor radii up to $300 \mathrm{pc}$. As main result of this work we show that the existing limits on CR anisotropies strongly restrict the contribution of the $\mathrm{CNO}$ element group to the Galactic $\mathrm{CR}$ component above $E \gtrsim 1 \mathrm{EeV}$, while the contribution of iron is restricted above $E \gtrsim 3 \mathrm{EeV}$.

Details of the method to generate turbulent magnetic fields and GMF models used are discussed [1]. Also we verified our numerical code by direct comparison with previous studies. In appendix of [1] it is shown that results of our simulations are in very good agreement with previous studies for same magnetic field structures. Results of numerical simulations are presented below in the Section 2.

\section{ANISOTROPY OF GALACTIC COSMIC RAYS PREDICTED AT EARTH AND NUMBER OF THEIR SOURCES}

We assume that the sources of Galactic CRs are located in a cylinder along the Galactic disc with height $z_{\max }$ and radius $r_{\max }$. In the following we assume $r \leq 20 \mathrm{kpc}$. Larger extensions of the source region along the $z$-direction would reduce the predicted anisotropy. We verified that, as long as the source region is less extended than $|z| \lesssim 500 \mathrm{pc}$, no strong modification of the results and conclusions would arise. We assume $|z|=200 \mathrm{pc}$ for most of the figures below. For each of the following plots we backtrack $10^{4}$ particles. We find that this induces an error $\leq \pm 3 \%$ on the predictions of the dipole amplitude. Reducing further this error to $\leq \pm 1 \%$ could in principle be achieved by backtracking $\simeq 10$ times more CRs, but it is in practice impossible due to computing time reasons.

Figures 1-2 present the simulated predictions for the dipolar amplitude at Earth for several Galactic CR primaries: protons, carbon (representative for the CNO group) and iron. CR sources are assumed to be located in the thin disk with $-200 \mathrm{pc} \leq z \leq 200 \mathrm{pc}$ and $r \leq 20 \mathrm{kpc}$. The recent model from ref. [16] is assumed for the regular GMF. Details of the model and it's parameters are discussed in ref. [1]. The turbulent field parameters (unless varied) are taken to be $B_{0}=4 \mu \mathrm{G}, z_{0}=2 \mathrm{kpc}, L_{\text {min }}=1$ AU and $L_{\max }=200$ pc. Details of possible profiles of tubulent field are discussed in ref. [1]. We include in these figures measurements of, and 99\% C.L. upper limits on, the dipole amplitude in right ascension from the Pierre Auger Collaboration, as indicated in the captions. We assume that the true dipole vector does not lie in the equatorial plane, so that these upper limits in right ascension do not overconstrain significantly the total amplitude. One cannot exclude the dipole amplitude for 1-few EeV Galactic iron to be below the Auger limits. In the Figures 1 and 2, the red dashed lines represent the lower error bars on the red shaded bands. These bands represent the allowed ranges for the dipole amplitude with Galactic iron primaries, for different turbulent GMF parameters.

Depending on the composition at $E \simeq 10^{18} \mathrm{eV}$, this has an important implication for the transition energy between Galactic and extragalactic CRs: For the set of GMF parameters assumed here, if the CR 

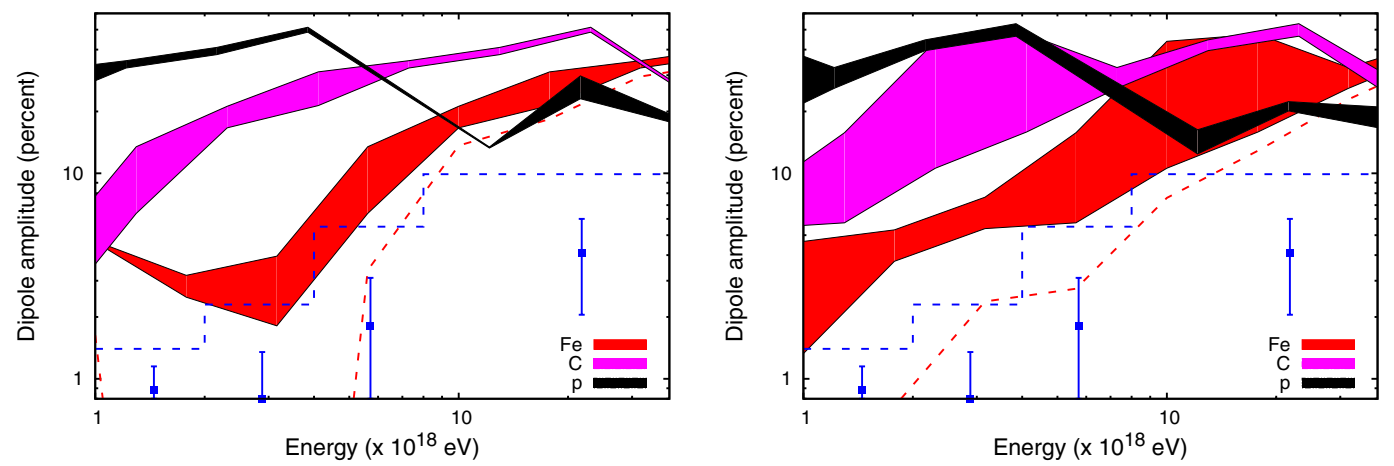

Figure 1. Dependence of the predicted dipole amplitude on the turbulent field spectral index and on its maximum spatial variation scale. The dashed blue line represents the $99 \%$ C.L. upper limit on the dipole amplitude in right ascension as measured by the Pierre Auger Observatory [18]. Blue points represents the Pierre Auger measurements of the dipole in right ascension with the "East-West method" for the 1-2 EeV bin and with the "Rayleigh analysis" for the three other energy bins, according to Fig. 5 of Ref. [18]. Left panel: shaded area for $\alpha \in$ [3/2,5/3] (from Kraichnan to Kolmogorov); Right panel: shaded area for $L_{\max } \in[100 \mathrm{pc}, 300 \mathrm{pc}]$. Red dashed lines for the lower error bars on the iron filled curve.
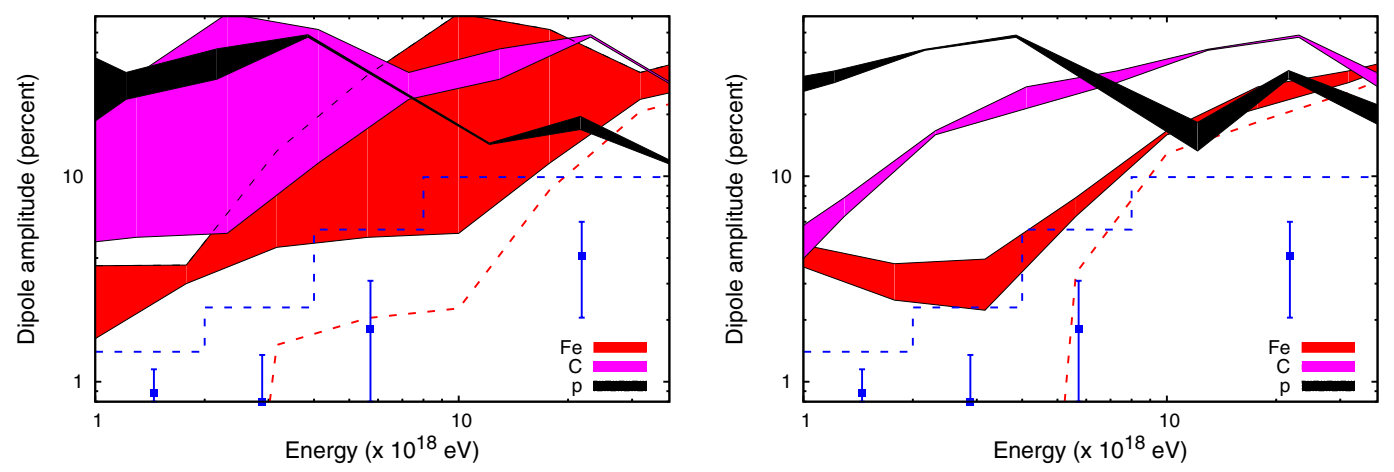

Figure 2. Dependence of the predicted dipole amplitude on the turbulent field strength at Earth and on its extension in the Galactic halo. For comparison, Pierre Auger data [18] are shown in blue. Left panel: shaded area for $B_{0} \in[2 \mu \mathrm{G}, 8 \mu \mathrm{G}] ;$ Right panel: shaded area for $z_{0} \in[2 \mathrm{kpc}, 8 \mathrm{kpc}]$. Red dashed lines for the lower error bars on the iron filled curve.

primary composition is predominantly light $(\mathrm{p})$ or intermediate $(\mathrm{C}, \mathrm{N}, \mathrm{O})$ at these energies, the predicted anisotropy at Earth would be larger than the 99\% C.L. upper limits from the Pierre Auger experiment if these nuclei were of Galactic origin. This implies that if the composition at $E \simeq 10^{18} \mathrm{eV}$ is measured to be light or intermediate, scenarios in which the transition from Galactic to extragalactic CRs occurs at the ankle are strongly disfavoured, at least for a wide range of GMF parameters.

Figures 1 and 2 present the dependence of the dipole amplitude on the turbulent GMF parameters, which are poorly constrained: the index $\alpha$ of the fluctuation spectrum (Fig. 1 - left panel), the maximal length of field fluctuations $L_{\max }$ (Fig. 1, right panel), the field strength normalization $B_{0}$ (Fig. 2, left panel) and the scale height $z_{0}$ (Fig. 2 - right panel). The shaded areas of the filled cures represent the relative change of the results for $\mathrm{p}, \mathrm{C}$ and $\mathrm{Fe}$ primaries when varying separately the four above parameters. The red dashed line is computed as the lower boundary of shaded areas for iron primaries minus 3\% from our statistical uncertainties.

At low rigidities $E / Z \lesssim 4 \mathrm{EeV}$, the dipole amplitude grows with $E / Z$ as expected. At larger rigidities, CRs start to enter the ballistic regime and higher order multipoles start to make a significant 

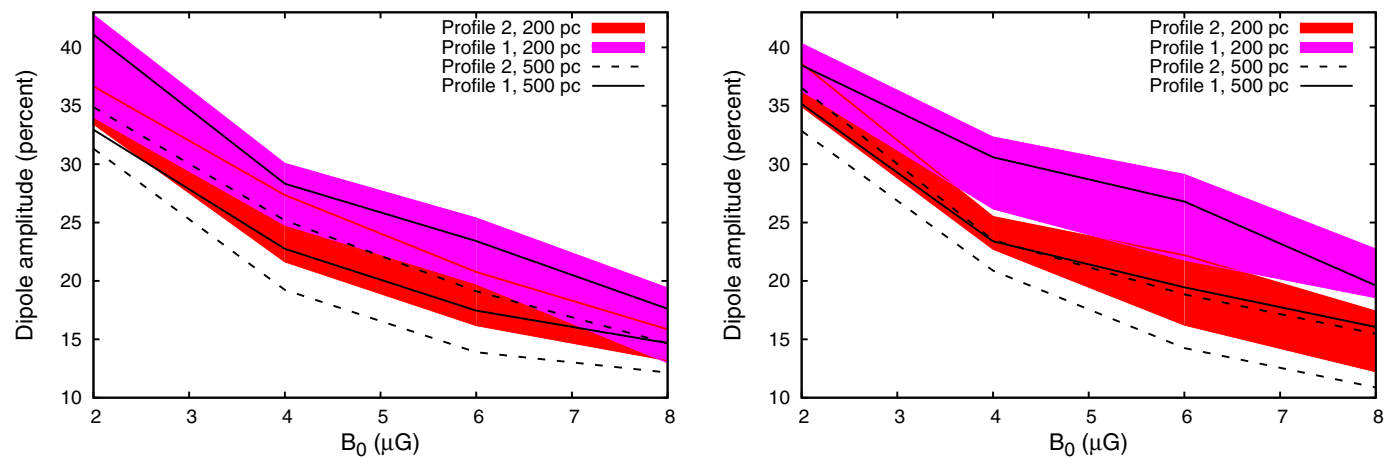

Figure 3. Predicted amplitude of the dipole versus the turbulent Galactic magnetic field strength, for $E / Z=$ $10^{18} \mathrm{eV}$ cosmic rays emitted by Galactic sources distributed in the region with $r \leq 20 \mathrm{kpc}$, and $-200 \mathrm{pc} \leq z \leq$ $200 \mathrm{pc}$ or $-500 \mathrm{pc} \leq z \leq 500 \mathrm{pc}$, respectively, as indicated. Profiles 1 and 2 for the turbulent Galactic magnetic field profile along $z$, as indicated. For the regular GMF the model from [16] is assumed. Shaded or delimited areas correspond to $z_{0}$ varying in the range $1-8 \mathrm{kpc}$. For the turbulent component a Kolmogorov (left panel) or Kraichnan (right panel) spectrum with $L_{\min }=1 \mathrm{AU}$ and $L_{\max }=200 \mathrm{pc}$ is assumed.

contribution to the total Galactic CR anisotropy at Earth. As seen in Figs. 1 and 2, the dipole amplitude may then become smaller and/or vary with $E / Z$. Thus a decrease of the dipole amplitude at high energies does not necessarily imply that the distribution of CR arrival directions at Earth becomes more isotropic.

The widths of the filled curves indicate that results mostly vary with the turbulent GMF strength and its maximum spatial variation scales. Results are less sensitive to the spectral index of the field and no strong difference in the dipole amplitude is found between the fields with Kolmogorov and Kraichnan spectra. The amplitudes at $E / Z \gtrsim 3 \mathrm{EeV} / 26$ are slightly larger for the Kraichnan spectrum because less power is present in the large length scale modes, which are relevant at such rigidities, than for the Kolmogorov spectrum. Results are only marginally sensitive to the extension $z_{0}$ of the turbulent field into the halo, see right panel of Fig. 2. This is due to the fact that CRs which escape the source region and propagate to large $z$ in the halo rarely come back to the source region.

Results are mostly sensitive to $L_{\max }$ and $B_{0}$. The maximal length scale of the turbulence $L_{\max }$ determines up to which energy CRs still scatter on the turbulent field inhomogeneities. For larger $L_{\max }$, CRs can be diffusive up to larger energies, which therefore reduces their expected anisotropy at Earth. One can see in the right panel of Figure 1 that for $L_{\max }=300 \mathrm{pc}$ and $B_{0}=4 \mu \mathrm{G}$, the dipole amplitude below $E \simeq 15 \mathrm{EeV}$ for a pure iron composition may be compatible with the current $99 \%$ C.L. upper limits from the Pierre Auger Observatory.

The dependence of our results on the turbulent field strength $B_{0}$ is very strong, see the left panel of Fig. 2. The upper parts of the shaded areas correspond to $B_{0}=2 \mu \mathrm{G}$ and the lower to $B_{0}=8 \mu \mathrm{G}$. For $B_{0}=8 \mu \mathrm{G}$, the anisotropy at Earth of iron primaries is a priori compatible with the Pierre Auger upper limits up to $E \simeq 20 \mathrm{EeV}$. For $B_{0}=2 \mu \mathrm{G}$, the dipole amplitude starts to overshoot the Pierre Auger limits around $E \simeq 3 \times 10^{18} \mathrm{eV}$, while for $B_{0}=4 \mu \mathrm{G}$, the amplitude starts to exceed the Pierre Auger limits around $10 \mathrm{EeV}$. For all cases, a light or intermediate composition at $E \simeq 10^{18} \mathrm{eV}$ would exceed the Pierre Auger upper limits and rule out the ankle as the transition from Galactic to extragalactic cosmic rays.

Figure 3 shows how the strength predicted at Earth of the dipole amplitude of $1 \mathrm{EeV}$ protons from Galactic sources depends on the turbulent field rms strength $B_{0}=2-8 \mu \mathrm{G}$ when the scale height $z_{0}$ is allowed to vary in the range 1 to $8 \mathrm{kpc}$ (shaded areas). Both turbulent field profiles are tested, and a $\pm 500 \mathrm{pc}$ width source region is also tested. Both for Kolmogorov (left panel) and Kraichnan spectra (right panel), predicted dipole amplitudes are above $10 \%$, considerably higher than the $\sim 2 \%$ upper limit 

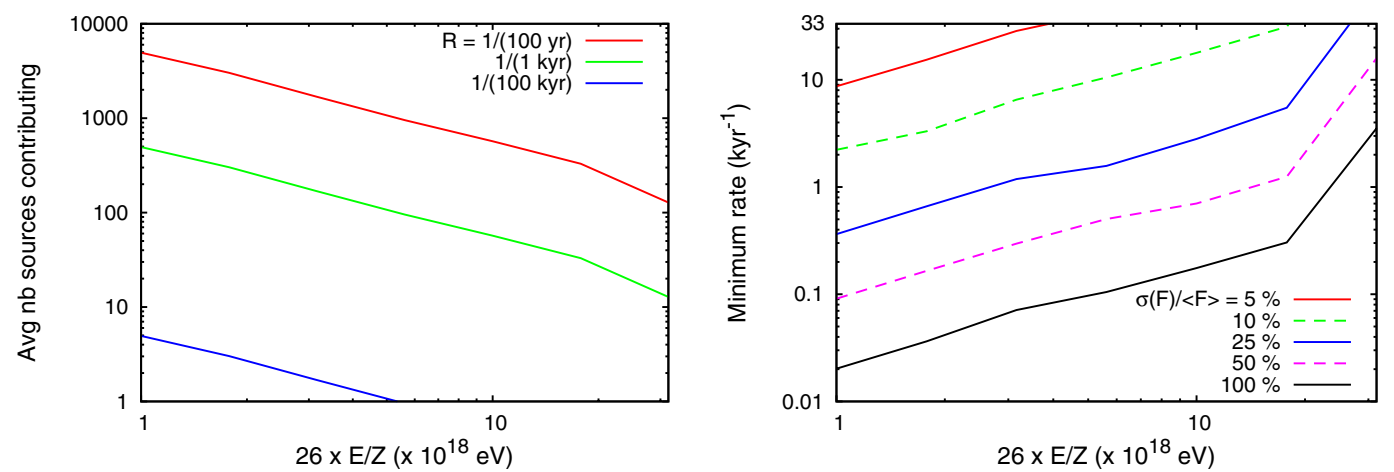

Figure 4. Left panel: estimate for the number of sources that would contribute to the Galactic CR flux at Earth versus $26 \times E / Z$, for three different source rates $\mathcal{R}=1 /(100 \mathrm{yr}), 1 /(1 \mathrm{kyr}), 1 /(100 \mathrm{kyr}) ;$ Right panel: estimate for the minimum rate $\mathcal{R}$ of Galactic $\mathrm{CR}$ sources that would be required to maintain relative fluctuations of the Galactic CR flux at Earth $\sigma(F) /\langle F\rangle$ below $\approx 5,10,25,50,100 \%$, versus $26 \times E / Z$.

from the Pierre Auger experiment at such energies. Therefore, the ankle cannot be the signature of the transition from Galactic to extragalactic CRs, if the contribution of Galactic protons to the CR flux at $1 \mathrm{EeV}$ is larger than $\simeq 20 \%$. Such a scenario would be consistent with the dip model [2]. A CR flux at $1 \mathrm{EeV}$ consisting mostly of light nuclei cannot be of Galactic origin, except in the very unlikely case of $B_{0} \gg 10 \mu \mathrm{G}$.

Let us now estimate more quantitatively when the continuous source distribution approximation breaks down. The average number of sources that would contribute to the Galactic CR flux at one given rigidity can be estimated as the average time spent by CRs in the source region multiplied by the source rate $\mathcal{R}$. We plot in Fig. 4 (left panel) this estimate of the number of contributing sources, for three different rates $\mathcal{R}=10,1,0.01 \mathrm{kyr}^{-1}$ and for CR rigidities in the range $E / Z=(1-32) \mathrm{EeV} / 26$. We assume here for the turbulent GMF a Kolmogorov spectrum with $L_{\max }=200 \mathrm{pc}$, strength $B_{0}=4 \mu \mathrm{G}$ and scale height $z_{0}=2 \mathrm{kpc}$. For sources with a rate $\mathcal{R}=1 \mathrm{kyr}^{-1}$ (green line) similar to that expected for magnetars, the average number of contributing sources stays above $\gtrsim 100$, up to the ankle for iron nuclei. It decreases from $\sim 500$ for $1 \mathrm{EeV}$ iron to only $\sim 10$ at $32 \mathrm{EeV}$. As shown below, $\sim 10$ sources is too small for the continuous source distribution approximation to be valid. With a ten times larger source rate $\mathcal{R}=10 \mathrm{kyr}^{-1}$ (red line), there would be $\gtrsim 100$ sources contributing for all the explored rigidity range, but $10 \mathrm{kyr}^{-1}$ is of the order of the Galactic supernovae rate which looks very unlikely for extreme CR accelerators. The blue line corresponds to rarer transients $\mathcal{R}=0.01 \mathrm{kyr}^{-1}$. At the ankle, only one source would contribute in average. Sources such as Galactic GRBs with $\mathcal{R} \sim 1 \mathrm{Myr}^{-1}$ cannot be described by the continuous source distribution approximation. However, such sources are unlikely to be responsible for the sub-ankle CR flux if it were to be of Galactic origin. Indeed, one can for example hardly match the bumpy CR spectrum resulting from rare Galactic transients to the observed smooth power law spectrum [17].

We provide in Fig. 4 (right panel) an estimate of the minimum rate $\mathcal{R}$ of Galactic sources that would be required to maintain relative fluctuations of the flux $\sigma(F) /\langle F\rangle$ below five given thresholds $(\approx 5,10,25,50,100 \%)$ on $\gg \mathcal{R}^{-1}$ time scales. To maintain $\sigma(F) /\langle F\rangle$ below $\approx 5 \%$ (red solid line) at the ankle for Galactic iron primaries, sources with rates comparable with that of Galactic supernovae $\mathcal{R} \approx 30 \mathrm{kyr}^{-1}$ would be needed. For rates $\mathcal{R} \sim 1 \mathrm{kyr}^{-1}, \sigma(F) /\langle F\rangle \approx 10 \%$ for $1 \mathrm{EeV}$ iron nuclei and remains $\lesssim 25 \%$ below the ankle. In this case, the continuous source distribution approximation is still valid. For energies $E \gtrsim(10-20) \mathrm{EeV}$, it quickly starts to break down: For $20 \mathrm{EeV}$ (resp. 30 EeV) iron nuclei, $\sigma(F) /\langle F\rangle \approx 50 \%$ (resp. $\approx 100 \%$ ). For Galactic source rates $\mathcal{R} \lesssim 0.01 \mathrm{kyr}^{-1}, \sigma(F) /\langle F\rangle$ exceeds $100 \%$ above $\sim 1 \mathrm{EeV}$ and the anisotropy measured at Earth is expected to significantly differ from the averaged values presented in the previous section. 


\section{EPJ Web of Conferences}

\section{CONCLUSIONS AND PERSPECTIVES}

In this work we studied the consistency of a transition from Galactic to extragalactic CRs with existing anisotropy limits as a function of energy above $E=10^{18} \mathrm{eV}$. The diffusion approximation becomes unreliable at $\mathcal{O}(E / Z) \sim 10^{16} \mathrm{eV}$, and therefore we studied the propagation of CRs in the Galactic magnetic field directly by backtracking trajectories. We simulated the turbulent magnetic field on nested grids which allows one to include turbulent field modes $B_{k}$ with arbitrary small wave-lengths. For the regular Galactic magnetic field we used up-to-date models from Ref. [16]. Because the global structure of the GMF is still rather uncertain, we studied the dependence of the resulting anisotropy on the magnetic field parameters such as its strength $B_{0}$, scale height $z_{0}$, correlation length $L_{\mathrm{c}}$ and exponent $\alpha$ of its power-spectrum. We also examined the dependence of our results on the width and height of the disk in which sources are located.

The main results of this study are presented in the Figs. 1-3. They show that the anisotropy mostly depends on the amplitude $B_{0}$ of the magnetic field in the disk. As our main conclusion from this study, we found that existing anisotropy limits are not compatible with light (proton) and intermediate (CNO) nuclei of Galactic origin as dominant contribution to the CR flux above $1 \mathrm{EeV}$. By contrast, Galactic iron nuclei as CR primaries are consistent with the existing limits even up to $10-20 \mathrm{EeV}$, if the strength of the turbulent field is as large as $B_{\mathrm{rms}} \sim 8 \mu \mathrm{G}$. This finding implies that determining the chemical composition of the CR flux around $10^{18} \mathrm{eV}$ settles also the question of the transition energy between Galactic and extragalactic component: As light nuclei at this energy are not sufficiently isotropized, they have to be extragalactic. Therefore the fast increasing proton contribution indicated by the KASCADE-Grande collaboration between $10^{17} \mathrm{eV}$ and $10^{18} \mathrm{eV}$ suggests the beginning of an extragalactic component.

We also studied qualitatively the dependence of the anisotropy on the effective density of sources, see Figs. 4. The average escape time of iron nuclei with $10 \mathrm{EeV}$ energy from the Galaxy is $\sim 10^{5} \mathrm{yr}$. Assuming for magnetars a rate of $10^{-3} / \mathrm{yr}$, the effective density of magnetars as sources of CR at $10 \mathrm{EeV}$ is $\sim 100 /$ Galaxy. Thus magnetars satisfy the anisotropy constraint and can be natural candidates for the sources of the high-energy end of the Galactic CR flux in the scenario where the transition from Galactic to extragalactic cosmic rays occurs at the ankle, provided they are able to accelerate iron up to few $\times 10^{18} \mathrm{eV}$.

In summary, we conclude that models with a transition from Galactic to extragalactic cosmic rays around the ankle are consistent with the existing anisotropy limits if the composition of Galactic cosmic rays at $E \gtrsim 10^{18} \mathrm{eV}$ is dominated by heavy nuclei. In contrast, if the chemical composition at these energies turns out to be light or intermediate, a transition at the ankle would be very strongly disfavoured.

\section{References}

[1] G. Giacinti, M. Kachelriess, D. V. Semikoz and G. Sigl, JCAP 1207 (2012) 031 [arXiv:1112.5599]

[2] V. S. Berezinsky and S. I. Grigor'eva, Astron. Astrophys. 199 (1988) 1; V. Berezinsky, A. Z. Gazizov and S. I. Grigorieva, Phys. Rev. D 74 (2006) 043005; astro-ph/0210095

[3] M. Lemoine, Phys. Rev. D 71 (2005) 083007 [astro-ph/0411173]

[4] K. Kotera and M. Lemoine, Phys. Rev. D 77 (2008) 023005 [arXiv:0706.1891]

[5] D. Allard, E. Parizot and A. V. Olinto, Astropart. Phys. 27 (2007) 61 [astro-ph/0512345]

[6] P. Sokolsky, "Final Results from the High Resolution Fly's Eye (HiRes) Experiment," PoS ICHEP 2010 (2010) 444 [arXiv:1010.2690]

[7] Y. Tsunesada [for the Telescope Array Collaboration], "Highlights from Telescope Array," arXiv: 1111.2507

[8] J. Abraham et al. [Pierre Auger Observatory Collaboration], Phys. Rev. Lett. 104 (2010) 091101 [arXiv:1002.0699] 


\section{UHECR 2012}

[9] P. Facal et al. [ The Pierre Auger Collaboration ], [arXiv:1107.4804]

[10] A. V. Glushkov et al, JETP Lett. 87 (2008) 190 [arXiv:0710.5508]

[11] W. D. Apel et al. [KASCADE-Grande Collaboration], arXiv:1111.5436 [astro-ph.HE]; see the contribution of A. Haungs, "Cosmic ray measurements with KASCADE-Grande"

[12] P. Blasi and E. Amato, JCAP 1201 (2012) 010 [arXiv:1105.4521]

[13] P. Blasi and E. Amato, JCAP 1201 (2012) 011 [arXiv:1105.4529]

[14] G. Giacinti et al, JCAP 1008, 036 (2010) [arXiv:1006.5416]

[15] G. Giacinti et al, Astropart. Phys. 35 (2011) 192 [arXiv:1104.1141]

[16] M. S. Pshirkov et al, Astrophys. J. 738 (2011) 192 [arXiv:1103.0814]

[17] M. Pohl and D. Eichler, Astrophys. J. 742 (2011)114 [arXiv:1108.2135]

[18] P. Abreu et al. [The Pierre Auger Collaboration], Astropart. Phys. 34 (2011) 627 [arXiv:1103.2721] 